\title{
Avoiding Complications During Insertion
}

\author{
Steve Hill
}

\begin{abstract}
The reduction of complications is an integral theme of VHP; complications can occur during or post insertion. This chapter examines insertion-related complications including arterial puncture, nerve damage, and air embolus. The relationship between catheter complications and insertion site is explored including peripheral vein, internal jugular, axillary, and femoral-placed CVADs. Methods for clinicians to reduce insertion-related complications are discussed, including thorough patient assessment with pre-assessment of vasculature using ultrasound, visualization aids, and real-time imaging during the insertion.
\end{abstract}

\section{Keywords}

Adverse events - Accidental arterial access Nerve injury · Air emboli · Complications Central venous catheter . Femoral catheter Axillary catheter $\cdot$ Jugular catheter Ultrasound

\subsection{Introduction}

There are several complications and injuries that can occur during insertion of a VAD including arterial puncture, nerve injury, air embolism, and infection. Arterial puncture of the insertion needle occurs in 3.7-12\%; more significant injury with advancement of dilator occurs in $0.1-1.0 \%$ (Kornbau et al. 2015). Nerve impingement or injury occurs in a small percentage of VAD access and is characterized by intense pain reported by the patient, electric-type shooting pain, and numbness not unlike arterial access. The arterial and nerve access must be differentiated, and when unintended, the access attempt should be immediately aborted. Air embolism is a complication that may occur during insertion or post insertion when any access point to a central vein is left open to air. Other complications of infection, phlebitis, and thrombosis are post insertional complications where signs and symptoms will occur days, weeks, or months after placement of the device.

\subsection{Arterial Access}

Arterial access may be accidental, as in the case of intended venous access, or intentional for arterial blood sampling or hemodynamic monitoring. Accidental arterial puncture is often easy to spot secondary to pulsatile blood flow but may be less

\author{
S. Hill $(\bowtie)$ \\ The Christie NHS Foundation Trust, Manchester, UK \\ Precision Vascular and Surgical Services Ltd, \\ Manchester, UK \\ e-mail: info@precisionvascular.co.uk; \\ steve.hill@christie.nhs.uk
}


easy to identify in the arteries in extremities or on a hypotensive critically ill patient (Kornbau et al. 2015). Signs and symptoms include:

1. Forceful or pulsatile flow

2. Pain expressed by the patient when applicable

3. Pallor if in an extremity occurring below the site of access

4. Paresthesia/numbness/tingling reported by the patient

5. Color difference in blood, lighter than venous blood

If there is uncertainty of arterial punctured vessel, a pressure transducer can be used to assess for venous/arterial waveform or arterial blood gas sample analyzed (Kornbau et al. 2015). Training, supervision, and demonstrated ability to identify and manage accidental arterial access are a requirement of inserters.

Following identification of arterial access, a determination must be made for management based on peripheral or central vein access and the ability to compress. Arterial access in the periphery is easily managed with digital compression-promoting coagulation. Depending on the patient's medical condition and use of anticoagulating medications, pressure is continuously applied for five or more minutes. Application of a tourniquet for subsequent cannulation may result in harm, loss of coagulation to the area, and additional bleeding/hematoma formation impacting nerves and structures in the region. Arterial access in the regions of the neck or chest should result in consultation by a vascular surgeon to safely ligate and mitigate any future bleeding.

Complications from arterial puncture include compromised airway from hematoma, cerebral ischemia, excessive bleeding, arteriovenous fistula, hemothorax, pseudoaneurysm, thrombus formation, and death (Dixon et al. 2017). Dixon et al. (2017) undertook a systematic review of the literature related to inadvertent arterial puncture and reviewed 80 cases to identify optimal management. Eight out of 44 cases that were man- aged by removal and compression at the site of injury were complicated by stroke or thrombus. The authors found that fewer complications occurred if the device was left in situ while preparations were being made for endovascular or surgical intervention. Serious complications can still occur with needle or guidewire- the authors suggest that the lack of case study reporting was due to patients potentially being managed by removal and pressure. Reflected in the cases reviewed as only 4 of the 80 cases included placement of needle or guidewire puncture only. Surgical intervention was considered optimal intervention for those patients who are fit for general anesthesia; endovascular repair was only marginally less successful and may be more appropriate in medial subclavian injury due to restricted surgical access.

There may be several reasons for arterial placement of catheters while using ultrasound. During cannulation of the vein using ultrasound, visualization of the needle tip may be confused with visualization of the needle shaft (Bowdle 2014). This may provide false reassurance as the clinician is watching the needle tip on the ultrasound screen, but the ultrasound beam is over a section of the needle shaft, and the needle tip is actually placed in the artery. Alternatively, the needle may be within the vein while being inserted under ultrasound but then inadvertently placed into the artery when placing the guidewire as at this point ultrasound is not being used (Bowdle 2014). Learning practitioners may be more prone to needle movement at this stage until their skills are polished. Holding the needle with the non-dominant arm while resting the arm on the patient may increase stability and reduces movement of the needle tip and the risk of it moving out of the vein.

\subsection{Vein Wall Injury}

Damage to the vein wall can occur during VAD insertion; significant injury can lead to venous tears. Bodenham et al. (2016) suggest that the longitudinal cell structure of a vein makes it more 
prone to linear tears (Bodenham 2006). Kornbau et al. (2015) notes another mechanism for this injury is that the guidewire becomes trapped against the vessel wall, and subsequent passage of the dilator causes the wire to bow and push up against the vessel wall, potentially resulting in a linear tear (Kornbau et al. 2015). In addition, the risk of perforation increases if a catheter lies against the wall of the vein at an angle of greater than $40^{\circ}$, and major intrathoracic injuries may even require cardiopulmonary bypass to achieve cardiovascular stability while the injuries are repaired (Kornbau et al. 2015).

\subsection{Nerve Injury}

Nerve injury can be insertion-related, such as a needle being advanced into a nerve branch during insertion, or post insertion such as hematoma causing neuropathic symptoms. The incidence of nerve injury is estimated to be $1.6 \%$, and resolution of neuropathic symptoms related to CVAD placement may take up to 6-12 months (Kornbau et al. 2015). Reports of phrenic nerve palsy following the insertion of an internal jugular CVC have been recorded; effects can be transient or may require surgical intervention (Ahn et al. 2012). Other sites include Brachial plexus, phrenic nerve, and median nerve or other upper arm nerves. Howes and Dell (2006) describe nerve injury that occurred following the insertion of a catheter for hemofiltration via the femoral vein. The patient was free from motor or sensory symptoms but complained of pain, which became severe. Limb perfusion was not affected, and peripheral pulses were intact. Nerve compression from the catheter was suspected; it was removed and replaced with an internal jugular catheter. The symptoms resolved almost immediately, and the patient did not suffer any lasting neurological deficits (Hows and Dell 2006). Zhao and Wang (2014) report an incidence of PICC-related nerve injury of $0.8 \%$ from a review of 739 cases; all of the injuries were related to brachial vein approach.

\subsection{Air Embolism}

Air embolism can occur when negative intrathoracic pressure draws air into the vein (Kornbau et al. 2015). This can occur at cannulation when disconnecting the syringe to insert the guidewire, when the dilator is removed from the Peel-Away sheath prior to advancing the catheter, by not priming the catheter according to manufacturer instructions, or leaving catheter clamps open. If air embolism does occur, the patient should be placed in the left decubitus position, localizing air in the right atrium and right lung and preventing emboli from entering the pulmonary arterial system, suggests Kornbau et al. (2015); the author adds that this maneuver is not effective in patients with abnormal anatomy. Case study option here.

\subsection{Different Veins and Associated Risks}

The axillary vein drains the cephalic, basilic, and brachial veins; it is closely accompanied by the axillary artery. Bodenham et al. (2004) look in detail at this approach, recording 200 cases and measuring various elements of the insertion and anatomical structures. The authors identified that moving more laterally, the axillary vein reduces in size and the depth of the vein increases. The mean length of right axillary catheters was $21.4 \mathrm{~cm}$ and 24.6 for catheters inserted on the left; axillary insertions were successfully inserted in $96 \%$ of cases. Complications included axillary arterial puncture in three cases $(1.5 \%)$ and transient neuralgia in two cases (1\%). Limitations of the technique were patients with increased BMI, due to decreased image quality, and difficulty passing guidewires and dilators was experienced with higher BMI patients because of the angle caused by deeper veins. Another limitation of this approach is misplacement of the guidewire, and the catheter occurred $15.5 \%$ and $12.9 \%$, respectively, illustrating that the insertion technique would benefit from fluoroscopy or ECG/navigation technology (Fig. 8.1). 


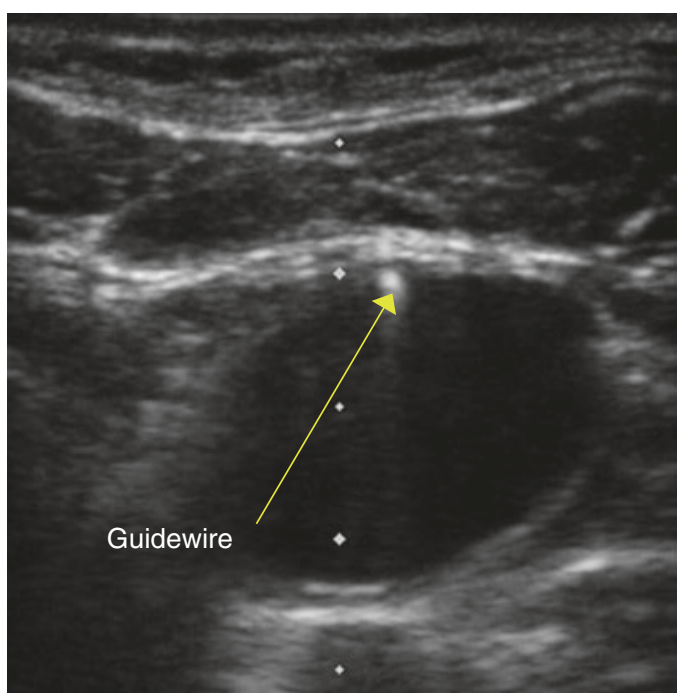

Fig. 8.1 Right internal jugular showing hyper-echogenic guidewire in position (used with permission of S. Hill)

Table 8.1 Incidence of complications for CVADs (Plumhans et al. 2011)

\begin{tabular}{l|l|l}
\hline Complication & Axillary/subclavian & Internal jugular \\
\hline Thrombosis & $3 \%$ & $0 \%$ \\
\hline Arterial puncture & 4 cases & 1 case \\
\hline $\begin{array}{l}\text { Dislocation } \\
\text { device }\end{array}$ & 1 case & 0 \\
\hline
\end{tabular}

\subsection{Axillary (Subclavian) Versus Jugular}

Plumhans et al. (2011) compared internal jugular versus axillary and subclavian vein insertions on 138 oncology patients and found that pain perception, radiation dose, tip migration, thrombosis, risk of arterial puncture, and dislocation of the device were all lower in the patients that received devices via the internal jugular vein (Plumhans et al. 2011). Plumhans et al. noted as follows: thrombosis, arterial puncture, and device dislocation rates for IJ and subclavian insertions. Conclusions from this publication were that IJ complications were less frequent (Table 8.1).

\subsection{Internal Jugular}

In a review of 123 consecutive patients who had a CT, the right internal jugular (RIJ) had an average diameter of $15.6 \mathrm{~mm}$ versus 11.7 left internal jugular, the overlap with the carotid artery was not significantly different comparing right to left, and depth of the skin to vein was also similar (Ozbek et al. 2013). The authors also examine the incidence of veins less than $7 \mathrm{~mm}$, which was $4.4 \%$ for RIJs and $21.9 \%$ for left internal jugular veins. Anatomy that presented outside of the expected landmarks occurred in $5.5 \%$ of cases, illustrating the importance of ultrasound and the limitation of landmark approach; human anatomy is not universal, and anatomical variations occur necessitating thorough ultrasound assessment, prior to the procedure and guidance during insertion (Lamperti et al. 2012; National Institute for Clinical Excellence 2002). Trendelenburg significantly increases the size of the internal jugular vein, increasing the safety of the procedure (Lewin et al. 2007). Parry (2004) assessed the influence of patient position upon the internal jugular approach and identified that the ideal position for the majority of patients was a $15 \%$ Trendelenburg position, with a small pillow

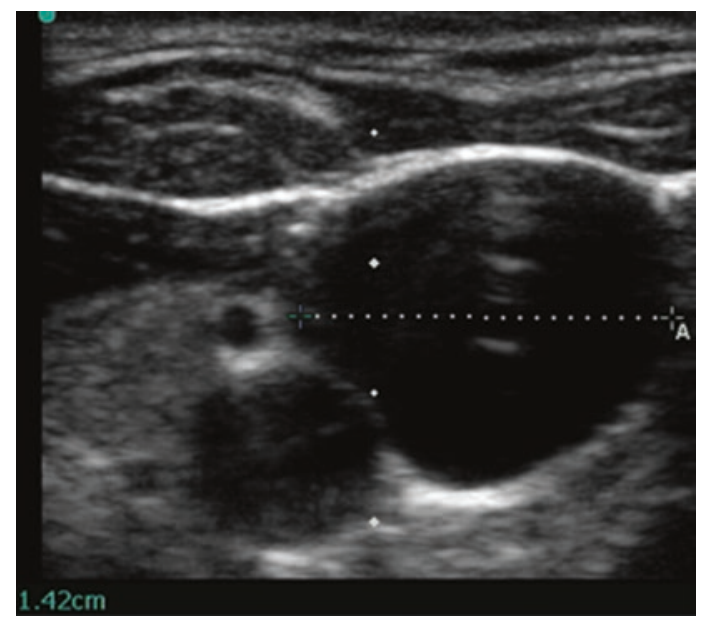

Fig. 8.2 Right internal jugular without Valsalva (used with permission of S. Hill) 


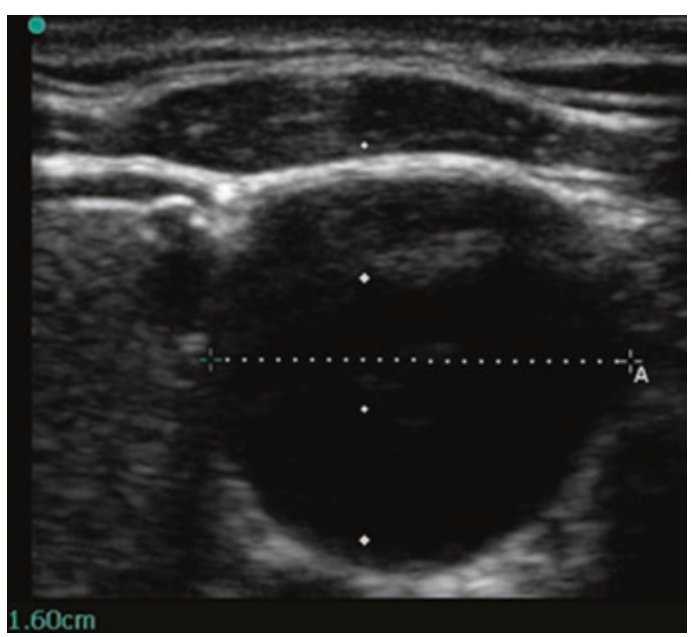

Fig. 8.3 Right internal jugular with Valsalva increase of $12.6 \%$ (used with permission of S. Hill)

under the head and with the head positioned close to the midline; however, positioning of the head close to the midline would be balanced against ease of access to the insertion site and effect on sterility of the procedure (Parry 2004).

The internal jugular was associated with the lowest mechanical complications compared with subclavian and femoral approaches using ultrasound (Tsotsolis et al. 2015).

Figures 8.2 and 8.3 show changes in the diameter of the RIJ with and without a Valsalva maneuver.

\subsection{Stenosis}

Once a catheter is placed, it can still have longerterm detrimental effects on the vein, inflammation, microthrombi, intimal hyperplasia, fibrotic changes, and stenosis (Agarwal 2009). Stenosis is not an immediate or always obvious complication of vascular access; patients may not always present with symptoms. It may only be identified when a further VAD is needed and either identified on ultrasound, a guidewire not passing, or by subtle enhancement of collateral vasculature. Yevzlin (2008) reviewed rates of stenosis in

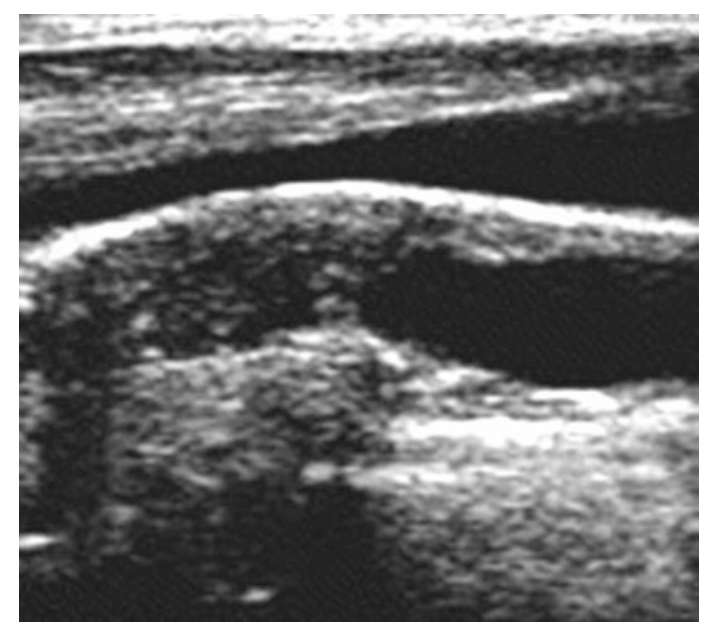

Fig. 8.4 Stenosis of the vein (used with permission of N. Moureau, PICC Excellence)

hemodialysis catheters and found from subclavian-placed catheters that stenosis rates varied from 40 to $50 \%$ and that comparative studies comparing 50 internal jugular-placed hemodialysis catheters to 50 subclavian-placed catheters saw $42 \%$ stenosis in the subclavian group versus $10 \%$ in those receiving internal jugular access (Fig. 8.4) (Yevzlin 2008). Gonsalves et al. (2003) looked at the 154 patients who underwent PICC insertions, and at the time of insertion, venography showed normal central veins (Gonsalves et al. 2003). Further evaluation showed that three patients developed central vein stenosis and that one patient developed central vein occlusion. From the 154 patients, 8 of the subsequent venograms showed vein occlusion and 10 patients had stenosis; a $7 \%$ incidence of stenosis or occlusion was found in patients with initial normal venograms. Patients in the study with longer dwell times were more likely to develop central vein anomalies. Related to the pathophysiological processes of PICC on vasculature, El Ters et al. (2012) looked at the associations between arteriovenous fistula function of 425 patients and found a strong independent association between PICC use and lack of functioning arteriovenous fistula (El Ters et al. 2012). Therefore, advice 
related to PICC for renal patients suggested by; the AV fistula first breakthrough initiative national coalition recommends NOT using PICC lines in patients at risk for, or with known midstage 3CKD, stage 4 and 5CKD or end-stage renal disease. A small-bore central catheter in the internal jugular vessels is recommended instead (Hoggard et al. 2008). Anatomical site and vein selection remain intrinsically linked to VHP and clinical outcomes, and it is essential to be aware of complications relevant to the selected site for CVAD insertion.

Wu et al. (2016) undertook a meta-analysis of percutaneous insertions internal jugular vs. subclavian vein implanted port placements and suggested that the internal jugular vein is a safer insertion site for implanted ports and is associated with lower risk mechanical complications (Wu et al. 2013).

To preserve patients' vasculature, INS standards (2016) include avoidance of placing PICCs in patients with chronic kidney disease due to the risk of central vein stenosis and occlusion (Gorski et al. 2016). The MAGIC recommendations quantify this and suggest PICCs should not be considered appropriate for patients with renal disease stage 3 or greater, with GFR of less than $44 \mathrm{~mL} / \mathrm{min}$, or for any patients receiving renal replacement therapy (Chopra et al. 2015). When considering the implication of stenosis from central venous catheterization, RCN guidelines recommend the internal jugular vein due to the lower relative risk of complication and suggest that the subclavian, external jugular, and femoral veins should be avoided due to the high risk of stenosis (RCN 2016).

\subsection{Femoral Approach}

Merrer et al. (2001) undertook an RCT that compared subclavian (144) vs. femoral site (145) insertions, mechanical complications differed, arterial puncture (AP) in the femoral group were higher, 13 in femoral versus 7 in the subclavian arm (Merrer et al. 2001). The APs in the femoral group led to two significant retroperitoneal hematomas that required blood transfusions or surgery.
Ultrasound scans were undertaken to detect the presence of thrombosis; in the subclavian group, thrombosis occurred in $1.9 \%$ patients and in $21.5 \%$ patients in the femoral arm, similar to thrombosis rates identified by Trottier (1995). Thrombosis was significantly higher in the femoral group at $21.5 \%$ versus 1.2 for the subclavian approach, seven patients in the femoral thrombosis group had complete catheter-related thrombosis, and pulmonary embolus was documented in two of the cases. Within this study they added that three patients would need to be treated using subclavian rather than femoral approach to prevent one complication. The authors looked at associations related to mechanical complications of insertion and found that there was an increased risk of mechanical complications associated with insertions that took place during the night, if the clinician was less experienced and if duration of insertion was prolonged.

Uhl and Gillot (2010) assessed 336 limbs to assess anatomical variations in femoral vein and found $12 \%$ with anatomical variations. Fronek et al. (2001) found the average width of the femoral vein to be $11.84 \mathrm{~mm}$ and that from the age of 60 years, size declined and velocity at rest significantly decreased over the age of 50 years (Fronek et al. 2001). Trendelenburg did not significantly improve femoral vein size but Valsalva maneuver was found to increase size by up to $40 \%$ (Lewin et al. 2007). Though mechanical risks exist, AAGBI guidelines suggest that in coagulopathic patients, the femoral approach may be advantageous, in the hands of an experienced clinician, as it allows easy compression of the site.

\subsection{Infection and Femoral Site}

Merrer et al. (2001) identified an incidence of infection for femoral insertions at 20 per/1000 and subclavian at 3.7 per/1000 cases and sepsis at $4.4 \%$ versus $1.5 \%$ (Merrer et al. 2001). Marik et al. (2012) undertook a systematic review looking at CRBSI risk or femoral subclavian and internal jugular catheters (Marik et al. 2012). The authors concluded that there was no significant difference between the infection rates of 
internal jugular and femoral sites in more recent studies and no difference between the three sites in terms of BSI (Marik et al. 2012). Timsit et al. (2013) looked at infection risk of jugular (1.0 per 1000 catheter days) versus femoral catheter (1.1 per 1000 catheter days) and found that femoral and internal jugular access leads to similar risk of infection, echoed by INS (2016) (Gorski et al. 2016; Timsit et al. 2013). Other factors with insertion leading to advantages of including femoral placement are tunneling techniques, moving the insertion site away from the inguinal fold with positioning toward the mid-thigh (Pittiruti 2014).

\subsection{Conclusion}

Acute care patients present with increasing comorbidities and complexities of illnesses. Clinicians must be aware, recognize, and subsequently safely manage insertion of the most appropriate intravenous devices to deliver treatment while avoiding complications to promote the best patient outcomes (Kornbau et al. 2015). While complications cannot be eliminated completely, prevention should be the ultimate goal.

\section{Case Study}

A 48-year-old male presented at the emergency room with severe shortness of breath, diaphoresis, low blood pressure, and lethargy. Following application of oxygen to the patient, the emergency department physician placed a right internal jugular catheter without ultrasound or visualization technology. The difficult insertion required three attempts. As the patient's adult-onset respiratory distress syndrome improved, he began complaining of chest pain, shortness of breath only when lying flat, and cough. During a sniff test of the diaphragm, right phrenic nerve paralysis was confirmed related to the insertion of the internal jugular catheter.

\section{Summary of Key Points}

1. Insertion-related complications can lead to profound implications for patients receiving CVADs.

2. An understanding of anatomy, thorough patient assessment, knowledge of patient history, use of ultrasound to assess the patient, and real-time imaging results in reduced complications.

3. Inserting clinicians must be able to recognize and manage CVAD-related complications.

4. Optimal patient outcomes for CVAD insertion requires the inserting clinician to be a vigilant, knowledgeable, and skilled practitioner; increased insertion attempts and clinician experience are closely linked to complication risk.

\section{References}

Agarwal AK. Central vein stenosis: current concepts. Adv Chronic Kidney Dis. 2009;16:360-70.

Ahn EJ, Baek CW, Shin HY, Kang H, Jung YH. Phrenic nerve palsy after internal jugular venous catheter placement. Korean J Anesthesiol. 2012;63:183-4.

Bodenham A, R Sharma A, Mallick A. Ultrasound-guided infraclavicular axillary vein cannulation for central venous access. Br J Anaesth. 2004;93(2):188-92. Epub, Jun 25.

Bodenham A. Ultrasound imaging by anaesthetists: training and accreditation issues. $\mathrm{Br} \mathrm{J}$ Anaesth. 2006;96:414-7.

Bodenham A, Babu S, Bennett J, et al. Association of Anaesthetists of Great Britain and Ireland: safe vascular access 2016. Anaesthesia. 2016;71(5):573-85.

Bowdle A. Vascular complications of central venous catheter placement: evidence-based methods for prevention and treatment. J Cardiothorac Vasc Anesth. 2014;28:358-68.

Chopra V, Flanders SA, Saint S, Woller SC, O'Grady NP, Safdar N, Trerotola SO, Saran R, Moureau N, Wiseman S, Pittiruti M, Akl EA, Lee AY, Courey A, Swaminathan L, Ledonne J, Becker C, Krein SL, Bernstein SJ. The Michigan Appropriateness Guide for Intravenous Catheters (MAGIC): results from a multispecialty panel using the RAND/UCLA appropriateness method. Ann Intern Med. 2015;163:S1-S40.

Dixon OG, Smith GE, Carradice D, Chetter IC. A systematic review of management of inadvertent arterial injury during central venous catheterisation. J Vasc Access. 2017;18:97-102. 
El Ters M, Schears GJ, Taler SJ, Williams AW, Albright RC, Jenson BM, Mahon AL, Stockland AH, Misra S, Nyberg SL, Rule AD, Hogan MC. Association between prior peripherally inserted central catheters and lack of functioning arteriovenous fistulas: a case-control study in hemodialysis patients. Am J Kidney Dis. 2012;60:601-8.

Fronek A, Criqui MH, Denenberg J, Langer RD. Common femoral vein dimensions and hemodynamics including Valsalva response as a function of sex, age, and ethnicity in a population study. J Vasc Surg. 2001;33:1050-6.

Gonsalves C, Eschelman D, Sullivan K, Dubois N, Bonn $\mathrm{J}$. Incidence of central vein stenosis and occlusion following upper extremity PICC and port placement. Cardiovasc Intervent Radiol. 2003;26:123-7.

Gorski L, Hadaway L, Hagle M, Mcgoldrick M, Orr M, Doellman D. Infusion therapy: standards of practice (supplement 1). J Infus Nurs. 2016;39:S1-S159.

Hoggard J, Saad T, Schon D, Vesely T, Royer T. Guideline for venous access in patients with chronic kidney disease: a position statement from the American Society of Diagnostic and Interventional Nephrology Clinical Practice Committee and the Association for Vascular Access. Semin Dial. 2008;21:186-91.

Howes B, Dell R. Risk of nerve damage from vascular access catheters, vol. 61. The Association Anaesthetists of Great Britain; 2006. p. 618.

Kornbau C, Lee KC, Hughes GD, Firstenberg MS. Central line complications. Int J Crit illn Inj Sci. 2015;5:170.

Lamperti M, Bodenham AR, Pittiruti M, Blaivas M, Augoustides JG, Elbarbary M, Pirotte T, Karakitsos $\mathrm{D}$, Ledonne J, Doniger S. International evidencebased recommendations on ultrasound-guided vascular access. Intensive Care Med. 2012;38:1105-17.

Lewin MR, Stein J, Wang R, Lee MM, Kernberg M, Boukhman M, Hahn I-H, Lewiss RE. Humming is as effective as Valsalva's maneuver and Trendelenburg's position for ultrasonographic visualization of the jugular venous system and common femoral veins. Ann Emerg Med. 2007;50:73-7.

Marik PE, Flemmer M, Harrison W. The risk of catheterrelated bloodstream infection with femoral venous catheters as compared to subclavian and internal jugular venous catheters: a systematic review of the literature and meta-analysis. Crit Care Med. 2012;40:2479-85.

Merrer J, De Jonghe B, Golliot F, Lefrant J, Raffy B, Barre E, Rigaud J, Casciani D, Misset B, Bosquet C, Outin H, Brun-Buisson C, Nitenenberg G, French Catheter Study Group in Intensive Care. Complications of femoral and subclavian venous catheterization in critically ill patients: a randomized controlled trial. JAMA. 2001;286:700-7.

National Institute for Clinical Excellence. NICE Technological Appraisal Guidance: guidance on the use of ultrasound locating devices for placing central venous catheters. NICE Technological Appraisal Guidance, 2002. p. 1-24.

Ozbek S, Apiliogullari S, Erol C, Kivrak AS, Kara I, Uysal E, Koplay M, Duman A. Optimal angle of needle entry for internal jugular vein catheterization with a neutral head position: a CT study. Ren Fail. 2013;35:492-6.

Parry G. Trendelenburg position, head elevation and a midline position optimize right internal jugular vein diameter. Can J Anesth. 2004;51:379.

Pittiruti M. The "off-label” use of PICCs. In: Sandrucci S, Mussa B, editors. Peripherally inserted central venous catheters. Milan: Springer; 2014.

Plumhans C, Mahnken AH, Ocklenburg C, Keil S, Behrendt FF, Günther RW, Schoth F. Jugular versus subclavian totally implantable access ports: catheter position, complications and intrainterventional pain perception. Eur J Radiol. 2011;79:338-42.

RCN. Standards for infusion therapy. 4th ed. London: Royal College of Nursing; 2016. p. 1-94.

Timsit J-F, Bouadma L, Mimoz O, Parienti J-J, Garrouste-Orgeas M, Alfandari S, Plantefeve G, Bronchard R, Troche G, Gauzit R. Jugular versus femoral short-term catheterization and risk of infection in intensive care unit patients. Causal analysis of two randomized trials. Am J Respir Crit Care Med. 2013;188:1232-9.

Trottier S, Veremakis C, O'Brien J, et al. Femoral deep vein thrombosis associated with central venous catheterization; results from a prospective randomised controlled trial. Crit Care Med. 1995;23:52-9.

Tsotsolis N, Tsirgogianni K, Kioumis I, Pitsiou G, Baka S, Papaiwannou A, Karavergou A, Rapti A, Trakada G, Katsikogiannis N. Pneumothorax as a complication of central venous catheter insertion. Ann Transl Med. 2015;3(3):40.

Uhl J, Gillot C. The foot venous pump: anatomy and physiology. Monaco: XVI World Congress of the Union Internationale de Phlebologie; 2009.

Uhl F, Gillot C. Anatomical variation of the femoral vein. J Vasc Surg. 2010;52(3):714-9.

Wu S, Ling Q, Cao L, Wang J, Xu M, Zeng W. Realtime two-dimensional ultrasound guidance for central venous cannulation: a meta-analysis. J Am Soc Anesthesiol. 2013;118:361-75.

Yevzlin AS. Hemodialysis catheter-associated central venous stenosis. Semin Dial. 2008;21(6):522-7.. Wiley Online Library

Zhao L, Wang Y. A descriptive study of nerve injury related to upper arm PICC placement. WoCoVa presentation. Zhejiang University School of Medicine; 2014. 
Open Access This chapter is licensed under the terms of the Creative Commons Attribution 4.0 International License (http://creativecommons.org/licenses/by/4.0/), which permits use, sharing, adaptation, distribution and reproduction in any medium or format, as long as you give appropriate credit to the original author(s) and the source, provide a link to the Creative Commons license and indicate if changes were made.

The images or other third party material in this chapter are included in the chapter's Creative Commons license, unless indicated otherwise in a credit line to the material. If material is not included in the chapter's Creative Commons license and your intended use is not permitted by statutory regulation or exceeds the permitted use, you will need to obtain permission directly from the copyright holder. 